\title{
To Keep COVID-19 Out of Hospitals in India - Are We Prepared?
}

\author{
Akanksha Ram Nibudey ${ }^{1}$, Vidya Sudhindra Baliga² ${ }^{2}$ Prasad V. Dhadse ${ }^{3}$ \\ ${ }^{1}$ Department of Periodontics, Sharad Pawar Dental College, Sawangi (Meghe) Datta Meghe Institute of Medical \\ Sciences (Deemed to Be University), Wardha, Maharashtra, India. ${ }^{2}$ Department of Periodontics, Sharad Pawar \\ Dental College, Sawangi (Meghe) Datta Meghe Institute of Medical Sciences (Deemed to Be University), Wardha, \\ Maharashtra, India. ${ }^{3}$ Department of Periodontics, Sharad Pawar Dental College, Sawangi (Meghe) Datta Meghe \\ Institute of Medical Sciences (Deemed to Be University), Wardha, Maharashtra, India.
}

\section{ABSTRACT}

\section{BACKGROUND}

The current viral outbreak of the Novel Corona virus, or COVID-19 has turned into a global health crisis. The World Health Organization (WHO) has declared it a public health emergency of international concern. Hospitals are known to have played a very important role in giving necessary medical treatment to the society, particularly at the time of a disaster. Preventive measures such as masks, hand hygiene practices, avoidance of public contact, case detection, contact tracing, and quarantines have been discussed as ways to reduce transmission. During the current outbreak of COVID-19, a break in these vital modalities and goods would lead to a break in the provision of acute health care by a not fully prepared health-care facility. Adding to it an elevated rate of personnel absence is predictable. A scarcity of important equipment and goods could prevent access to desired care and have a direct impact on health care delivery. Health workers and carers are at high risk of infection, and healthcare associated amplification of transmission is of concern as is always the case for emerging infections. Non-pharmaceutical interventions remain central for management of COVID-19 because there are no licensed vaccines or coronavirus antivirals. To date, effective treatment is lacking; however, clinical trials investigating the efficacy of several agents, including remdesivir and chloroquine, are underway in China. Currently, effective infection control intervention is the only way to prevent the spread of the infection. Considering this, India has issued several notifications on how one can be affected by this deadly disease, what the symptoms are, and what preventive measures could be adopted. In addition, the Government of India has issued guidelines for prevention and preparedness of hospitals. This review is to better understand the epidemiology, causes, clinical diagnosis, prevention and control of this virus and steps which are to be followed to prevent spread of disease in hospitals.

\section{KEY WORDS}

Preparedness of Hospitals in India, Hospital Management, Precautions, Personnel Protective Equipment (PPE)
Corresponding Author: Dr. Akanksha Ram Nibudey, Department of Periodontics, Sharad Pawar Dental College, Sawangi (Meghe) Datta Meghe Institute of Medical Sciences (Deemed to Be University), Wardha, Maharashtra, India E-mail: akankshanibudey16@gmail.com

DOI: $10.14260 / j e m d s / 2020 / 597$

How to Cite This Article: Nibudey AR, Baliga VS, Dhadse PV. To keep COVID-19 out of hospitals in India - are we prepared? J Evolution Med Dent Sci 2020;9(37):2751-2754, $10.14260 /$ jemds/2020/597

Submission 02-05-2020, Peer Review 01-08-2020, Acceptance 07-08-2020, Published 14-09-2020.

Copyright (C) 2020 JEMDS. This is an open access article distributed under Creative Commons Attribution License [Attribution 4.0 International (CC BY 4.0)] 


\section{BACKGROUND}

The SARS-CoV 2 or the 2019 novel corona virus has been showing a fast spread to the whole world from its place of first occurrence in Wuhan City of Hubei Province in China. ${ }^{1}$ Till 07/04/2020 around 1,381,014 patients of corona virus 2019 disease and 78,269 deaths have been confirmed. ${ }^{2}$ India has stated death of 124 cases till date out of 4,789 cases. Very few or rare cases are reported in children fortunately so far with no deaths; but as the future course of this virus is unknown, specially the hospitals should take necessary steps and follow some iconic protocols so as to avoid hospital spread of COVID19 in the hospitals themselves. This article gives a brief review about the protocols that should be strictly implemented by the hospitals for limiting the spread of the disease in hospital environment.

\section{Epidemiology}

The disease commencement of the 1st positive incident of 2019-nCoV virus was on 01/12/2019 in Wuhan, China. Originally an epidemic including a neighbourhood Huanan Seafood Market with at most 41 natives was stated. ${ }^{3}$ The native medical providers stated an "epidemiologic alert" on $31 / 12 / 2019$, and the market was sealed on $01 / 01 / 2020$. An overall 59 suspected patients with fever and dry cough were sent to a selected hospital. Of the 59 suspected, 41 patients were positive for the disease, detected by next generation sequencing or real-time reverse transcription-polymerase chain reaction. 27 patients had visited Huanan Seafood Market, ${ }^{3}$ though it seems to have a stipulation that the $1 \mathrm{st}$ patient on $1^{\text {st }}$ of December had not shown any record of Huanan Seafood Market visit and the consequent patient began to report only on $10^{\text {th }}$ of December, 9 days later. In the subsequent days, a spurt of patients were scattering from Wuhan to the whole Hubei province. The first transferred patient was into Thailand on January 13, 2020.

The WHO stated a public health emergency of global worry alarm on $30^{\text {th }}$ of January. The first death toll for cases in the hospital was predicted to be $11 \%-15 \%$. It is possible that person-to-person transmissions occur via droplets and contact. It seems that Nosocomial infections in the healthcare facilities occur and hence proper infection control is necessary. ${ }^{4-5}$ The WHO strategy of containment for elimination could be adjusted to include mitigation strategies combined with the following activities currently recommended on the WHO website:

1. Close monitoring is needed of changes in epidemiology and of the effectiveness of public health strategies and their social acceptance.

2. Continued evolution is needed of enhanced communication strategies that provide general populations and vulnerable populations who are at most risk, with actionable information for self-protection, including identification of symptoms, and clear guidance for treatment seeking.

3. Continued intensive source control is needed in the epicentre in China i.e. isolation of patients and persons testing positive for COVID-19, contact tracing and health monitoring, strict health facility infection prevention and control, and use of other active public health control interventions with continued active surveillance and containment activities at all other sites where outbreaks are occurring in China.

4. Continued containment activities are needed around sites outside China where there are infected people and transmission among contacts. Intensive study to provide information on transmissibility, means of transmission, and natural history of infection, with regular reporting to WHO and sharing of data.

5. Intensified active surveillance is needed for possible infections in all countries using the WHO recommended surveillance case definition.

6. Preparation for resilience of health systems in all countries is needed, as is done at the time of seasonal influenzaanticipating severe infections and course of disease in older people and other populations identified to be at risk of severe disease.

7. If widespread community transmission is established, there should then be consideration of a transition to include mitigation activities, especially if contact tracing becomes ineffective or overwhelming and an inefficient use of resources. Examples of mitigation activities include cancelling public gatherings, school closure, remote working, and home isolation, observation of the health of symptomatic individuals supported by telephone or online health consultation, and provision of essential life support such as oxygen supplies, mechanical ventilators and extracorporeal membrane oxygenation (ECMO) equipment.

8. Serological tests need to be developed that can estimate current and previous infections in general populations.

9. Finally, continued research is important to understand the source of the outbreak by study of animals and animal handlers in markets to provide evidence necessary for prevention of future coronavirus outbreaks. ${ }^{4}$

Considering the spread in India, Union Ministry of Health and Family Welfare has given a checklist for allotment of residents/PG students and nursing students as part of hospital management of COVID-19.5 The hospital may be separated into three large areas namely:

1. Non-Covid area.

2. Area to keep cases having mild to moderate sickness Covid area.

3. Critical area like the ICU and an area to keep cases having acute or severe respiratory illness. This area is required to be developed in the emergency room where cases are to be kept. 6

\section{Training of Residents}

A co-ordination is needed between the group giving instructions and the COVID-19 Task Force which is in charge for operation of residents. It is important to note that every resident is well trained before any COVID-19 duty is assigned by the hospital infection control team. Considering the three areas of facility, residents are trained according to the needs. So, when training is done at two places then same training 
should be given to the resident on duty. That causes a uniformity in the training given. The head controlling these facilities will be responsible to conduct the same level of training. Residents not under regular duties in COVID-19 ward should also be given information about clinical evaluation of patients. This program should be conducted by COVID-19 Task Force. We can also consider psychologists to be in it, to improve enthusiasm of residents' participation. ${ }^{7}$

\section{Faculty Deployment}

The similar common steps to categories department (depending upon medical contact and contribution in the managing of seriously sick cases) and level of facility should be used for deployment of faculty for this purpose.

\section{Private Hospitals / Colleges}

Similar principles may be used to private institutions also.

\section{Dental Students}

Similar principles should be given to dental doctors if there is a need. They have to be educated in infection control starting from now.

\section{Preparation Rules from an Indian Scenario}

Knowing the risk of transfer of coronavirus in India, these steps should be followed to prevent the spread of disease.

- Health care personnel should show special concern on the international travel history of patient in the previous two weeks. They should also note any respiratory symptoms and, should also ask about any contact history of this patient to any ill patients having international travel history.

- $\quad$ Wearing of N95 or surgical masks along with PPE should be done by health care personnel during examination of patients along with frequent follow-up of hand hygiene measures.

- For patients having respiratory illness, a triage system has to be made available in the outpatient department along with availability of surgical masks to such patients.

- History should be taken of patients about presence of diabetes or hypertention..$^{8-9}$

- To rule out whether the suspected patients are positive or not they should be sent to government designated centres for quarantine and testing.

- To prevent person to person spread and droplet spread of the disease from patients having symptoms of severe pneumonia and acute respiratory distress syndrome, they should be kept in an isolation ward.10

- Regular disinfection of the surfaces with $0.05 \%$ sodium hypochlorite is to be followed. Regular testing of the patients with multiplex PCR panels should be done and if found negative they should be further checked for severe acute respiratory syndrome corona virus. ${ }^{2}$

- All healthcare personnel need to remain informed about new improvements in global spread of the disease and preventive protocols. ${ }^{11}$

\section{DISCUSSION}

Health care personnel are at highest risk from danger of infection of COVID 2019. In the SARS-CoV 2 outbreak of 2002, healthcare personnel accounted for $21 \%$ of those suffered. The doctor who first reported about the virus has died. Up to $10 \%$ of the reported cases in China and up to $9 \%$ of all cases in Italy have been among healthcare workers. It is imperative to guard healthcare personnel to guarantee continuity of treatment and to check spread of contamination to new cases. ${ }^{12}$ From an online survey conducted including 410 IAS officials by means of the Government of India, it was observed that there is a critical scarcity in healthcare organization in our country to fight against this disease. Statements of the Union Ministry of Health and Family Welfare that there were ample beds, ventilators and PPE in India was proven false by this survey. It was found that adequate ventilators were not present which was told by $71 \%$ of the total respondents out of 266 . Starting from March $25^{\text {th }}-30^{\text {th }}, 2020$ the survey was carried out amongst district collectors and IAS officials. About 50\% claimed of insufficient PPE availability for health care personnel. "PPE like masks, gloves etc. meant for health care personnel remained a topic of worry for some regions and states," through the survey. Among the respondents $80 \%$ claimed that there is local body functioning to recognize, investigate and isolate infected patients. Whereas in some regions limited availability of testing kits was found. It was found that $60 \%$ of the respondents said that hospitals were not satisfactorily prepared.

'The COVID-19 Preparedness Survey' recognized the requirement to improve hospital preparedness in India. The sub-district hospitals and health centres' readiness is a cause of concern mainly in the Northeastern region. Along with capacity building and training for COVID-19 amongst healthcare personnel at district level.

\section{CONCLUSIONS}

This study shows a complete representation of the present protocols followed in hospitals in retort to the outbreak of COVID-19. Till now many research papers have been written about the origin and spread of disease and various possible sources and researches exploring avoidance and actions to prevent spread are being conducted. Indian government is making quick decisions to combine latest scientific results to community guidelines at the public, provincial and countrywide stages so as to decrease and / or avoid the advance spread of the COVID-19. At-home testing and monitoring of possible COVID-19 cases could ease pressure on the hospitals and emergency services and prevent the spread of infection. Under the proposed protocol, people suspected to have COVID-19 would be assessed, swabbed and monitored at home by specially trained paramedics in protective equipment, rather than being tested in hospital. More research and training is needed to deliver effective and consistent methods to achieve control over this crisis in both the short term and long term. 


\section{REFERENCES}

[1] Wang C, Horby PW, Hayden FG, et al. A novel coronavirus outbreak of global health concern. Lancet 2020;395(10223):470-3.

[2] Coronavirus Outbreak. https://www.worldometers. info/coronavirus/. Accessed 23 Feb 2020.

[3] Huang C, Wang Y, Li X, et al. Clinical features of patients infected with 2019 novel coronavirus in Wuhan, China. Lancet 2020;395(10223):493-506.

[4] World Health Organization. 2019-nCoV situation report. https://www.who.int/emergencies/diseases/novelcoronavirus 2019/situationreports/.

[5] Deployment of residents in various facilities designated for screening and management of patients with COVID-19 and the non covid area of the hospital (in this SOP, the term "resident" includes DNB and CPS students). https://gacs.world/CMS/govtMain/4323.

[6] Balwani M, Goswami J, Patel M. Intensive care unit scoring systems and outcome of CKD patients admitted in intensive care unit: a single center study. Nephrology Dialysis Transplantation 2017;32(3):iii259.

[7] Padole, Divyani, Kelkar P, et al. Stigma related to psychiatric disorders among physiotherapy students in central India. Indian J Psychiatry 2019;61(9)3:S495.

[8] Taksande A. A New scoring system for the diagnosis of pulmonary arterial hypertension. European Heart J 2017;38(1):158-59.

[9] Acharya S, Shukla S, Wanjari A. Subclinical risk markers for cardiovascular disease (CVD) in metabolically healthy obese (MHO) subjects. J Clin Diagn Res 2019;13(6):OC16.

[10] Dhar R, Singh S, Talwar D, et al. Bronchiectasis in India: results from the European multicentre bronchiectasis audit and research collaboration (EMBARC) and respiratory research network of India registry. Lancet Global Health 2019;7(9):E1269-79.

[11] Singhal T. A review of coronavirus disease-2019 (COVID19). Indian J Pediatr 2020;87(4):281-6.

[12] Chang D, Xu H, Rebaza A, et al. Protecting health-care workers from subclinical coronavirus infection. Lancet Respir Med 2020;8(3):e13. 